\title{
Filtered Intersections and Filtered Products
}

\author{
Nicholas Roersma \\ Mathematics and Computer Science Department \\ Wabash College \\ Crawfordsville, Indiana 47933-0352 USA
}

Received: September 10, 2002

Accepted: December 6, 2002

\begin{abstract}
We explore the filtered intersections and filtered products of ideals, modules, and other properties of commutative rings with zero divisors. Set theoretic properties of orderings are considered. The focus is then turned to the compliment of filters, ideals, and similar topics considered for ideal intersections.
\end{abstract}

\section{INTRODUCTION}

The investigation of properties under filtered intersections and filtered products is thoroughly explored in model theory [1]. The strong results, most particularly an extension of the Łos' Theorem, can be found in [2] and require a rigorous understanding of logic and model theory. These results are out of reach in most undergraduate settings, but the concept of the filtered intersection and filtered product are easily understood in the context of already learned definitions and properties. In this paper we investigate specific properties of rings and sets, particularly those not expressible in firstorder language. The focus of the paper is neither logic nor model theory, but the results of specific properties in relation to filtered intersections and filtered products without use of Łos' Theorem [3].

Let $R$ be a commutative ring with unity throughout the paper. When defining any set we will assume it is nonempty unless otherwise stated. Let $P(X)$ represent the power set of a set $X$, i.e., the set of all subsets of $X$.

Definition 1.1. Let $X$ be a nonempty set and let $D \subset P(X)$. We say that $D$ is a filter if it is nonempty and

(i) $\varnothing \notin D$

(ii) $A, B \in D$ implies $A \cap B \in D$.

(iii) $A \in D$ and $A \subset B$ implies $B \in D$.
$D$ is called an ultrafilter on $X$ if it is a filter and for an arbitrary $A \in P(X)$ we have either $A \in D$ or $X \backslash A \in D$.

$D$ is called a principal filter on $X$ if $D=\{B \in P(X) \mid A \subseteq B\}$ for some fixed $A \in$ $P(X)$. Here, $A$ is called the base element of $D$. If $A=\{a\}$, then $D$ is said to be a singleton.

Property (iii) of a filter is known as being "closed upward." There are strong relations among different types of filters. The following results form a basic understanding of filter properties.

Lemma 1.2. Let $X$ be a finite set. If $D$ is a filter on $X$, then $D$ is a principal filter.

Proof. Since $X$ is finite, so is $P(X)$. Thus, every element of $D$ and $D$ itself has finite cardinality. Let $m=\min \{|A| \mid A \in D\}$. Let $|P|,|C|=m$ where $P, C \in D$. Here $\varnothing \neq$ $P \cap C \in D$. If $P \neq C$, then $|P \cap C|<|P|=C$, a contradiction. This implies that $P$ is the unique element of $D$ with cardinality $m$. Consider $A \in D$. Again, $\varnothing \neq A \cap P \in D$ and $A \cap P \leq|P|$. If $P \not \subset A$, the $|A \cap P|<|P|$ since $P$ is finite, but this is a contradiction of the assumption of $P$ being of minimal cardinality. Thus, $|A \cap P|=|P|$ and $P$ is finite, so $A \cap P=P$, yielding $P \subseteq A$. Since $A$ is an arbitrary element of $D$, we have that $D$ $=\{A \mid P \subseteq A\}$, a principal filter. 
The following results can be found in [2] or easily verified by the reader. These results will be used throughout the paper.

Lemma 1.3. If $X$ is an infinite set, then there exists an ultrafilter $D \subset P(X)$ that is not a principal filter on $X$.

Lemma 1.4. Given a set $X$, a principal filter $D$ on $X$ is an ultrafilter if and only if $D$ is a singleton.

Lemma 1.5. Let $X$ be a finite set. If $D$ is an ultrafilter on $X$, then $D$ is a singleton.

Corollary 1.6. Let $X$ be an infinite set. If $D$ is an ultrafilter on $X$, then $D$ is a singleton or every element of $D$ has infinite cardinality.

\section{FILTERED INTERSECTIONS}

Definition 2.1. Let $I$ be an index, $D$ be a filter on $I$, and $A_{i}$ be a set for every $i \in I$. The set $\left\{a \mid\left\{i \mid a \in A_{i}\right\} \in D\right\}$ is called the filtered intersection of the $A_{i}$ with respect to $D$, denoted $\prod A_{i} / D$.

Here we use the definition of the filter to select which elements of our indexed sets will become elements of our final set, the filtered intersection. Given any principal filter on the indexing set, the elements present in the filtered intersection are entirely determined. The following theorem will be used continually throughout this section to create simple examples and counterexamples.

Theorem 2.2. Let I be an arbitrary indexing set for nonempty sets $A_{i}$ where $i \in I$. If $D$ is a principal filter on $I$ with a base element $P$ having finite cardinality, then the filtered intersection $A=\prod A_{i} / D=\cap_{i \in P} A_{i}$.

Proof. Define $C=\cap_{i \in I} A_{i}$. Define a subset of $C, B=\left\{\mathrm{r} \mid \mathrm{R} \in A_{i}\right.$ for all $\left.i \in P\right\}=\cap_{i \in P} A_{i}$. By assumption, $P \in D$, so for any $r \in B, r \in$ $A$. Consider $s \in C \backslash B$. There exists a $j \in P$ such that $s \notin \mathrm{A}_{\mathrm{j}}$. Then, where $S=\left\{\mathrm{i} \mid s \in A_{i}\right\}$, $j \notin S$. This implies $P \not \subset S, S \not \subset D$, and $s \notin A$. Therefore, $A=B=\cap_{i \in P} A_{i}$.

Theorem 2.2 provides the following corollary that provides examples more easily.
Corollary 2.3. Let $I$ be an arbitrary, finite indexing set for nonempty sets $A_{i}$ where $i \in$ I. If $D$ is a filter on $I$, then the filtered intersection $A=\prod A_{i} / D=\cap_{i \in P} A_{i}$ where $P$ is the base element of $D$.

Proof. From Lemma 1.2 we have that $D=$ $\{S \in P(I) \mid P \subseteq S\}$, a principal filter where $P$ is the base element. The rest follows from Theorem 2.2.

With the aid of Corollary 2.3, we find that any property shared by all of the $A_{i}$ may be preserved if we choose the filter correctly.

Corollary 2.4. Let $I$ be an arbitrary, finite indexing set for nonempty sets $A_{i}$ where $i \in$ I. Let $\Phi$ be a property and let $\Phi$ be true for $A_{i}$ for every $i \in I$. Then there exists a filter $D$ on $I$ such that $\Phi$ will be true for the filtered intersection $A=\prod A_{i} / D$.

Proof. Let $P=\{\alpha\}$ where $\alpha \in I$. Let $D$ be the principal filter with base element $P$. Here $A=A_{\alpha}$.

For the remainder of the paper the elements of the filtered intersection will not be of particular interest. Instead, the focus will be the relationship of the elements in the filtered intersection and their properties. We begin this investigation with properties of commutative rings familiar to most undergraduates with an algebra background.

Theorem 2.5. Let $R$ be a commutative ring with unity and $A_{i}$ be an ideal of $R$ where $i \in I$, an arbitrary indexed set. If $D$ is a filter on $I$, then the filtered intersection $A=\prod A_{i} / D$ is also an ideal of $R$.

Proof. Since $A_{i}$ is an ideal, $0 \in A_{i}$. Thus, $\left\{i \mid 0 \in A_{i}\right\}=I$ and $I \in D$. This implies $0 \in A \neq \varnothing$.

Let $\mathrm{a}, \mathrm{b} \in A$ and let $S=\{\mathrm{i} \in I \mid \mathrm{a}$ $\left.\in A_{i}\right\}$ and $T=\left\{j \in I \mid b \in A_{j}\right\}$. By definition of a filtered intersection $S, T \in D$. Consider $c=a-b \in R$. If $a, b \in A_{k}$, for a fixed $k \in I$, then by definition of an ideal, $c=a-b \in A_{k}$. Define $U=\left\{k \in I \mid a \in A_{k}\right.$ and $\left.b \in A_{k}\right\}$. 
Here, $U=S \cap T \in D$, and since $D$ is a filter, $U \neq \varnothing$. Let $V=\left\{l \in I \mid c \in A_{l}\right\}$ yielding $U \subseteq$ $V$. Again since $D$ is a filter and $U \in D, \vee \in$ $D$. Thus, $c \in A$. Consider ar, where $r \in R$. By the definition of an ideal, if $a \in A_{i}$, then ar $\in A_{i}$ also. Fix an arbitrary $r \in R$, and define $W_{r}=\left\{m \in I \mid\right.$ ar $\left.\in A_{m}\right\}$. This yields that $S \subseteq W_{r}$ and since $D$ is a filter and $S \in$ $D, W_{r} \in D$. Thus ar $\in R$ for any $r \in R$.

Therefore, $A$ is an ideal of $R$.

This general case guarantees that the filtered intersection of the ideals will be an ideal. When we combine this theorem with Corollary 2.3, we have the following corollary, though easily proved independently.

Corollary 2.6. Let $R$ be a commutative ring with unity. If $A$ and $B$ are ideals of $R$, then $A \cap B$ is an ideal of $R$.

Though ideals are preserved under filtered intersection, specific types of ideals are not necessarily preserved in a filtered intersection. The following example, using both Corollary 2.3 and Theorem 2.5, shows that the filtered intersection of prime ideals may not be a prime ideal, although it will be an ideal.

Example 2.7. The set of prime ideals of the ring $Z_{6}$ is $\{(2)$, (3) $\}$ where (2) $=\left\{r \cdot 2 \mid r \in Z_{6}\right\}$. If we index these as $P_{\alpha}$ and $P_{\beta}$, respectively, and let $D=\{\{\alpha, \beta\}\}$, then $\prod P_{i} / D=\{0\}$. In $\mathrm{Z}_{6}$, however, $3 \cdot 2=0$ and $3,2 \notin(0)$. Therefore, $\prod P_{i} / D$ is not a prime ideal.

Of particular interest is that the filtered intersection of prime ideals always will be prime given a specific type of filter on the indexing set. The following theorem shows that an ideal ultrafilter for a filtered intersection of prime ideals is sufficient to yield a prime ideal.

Theorem 2.8. Let $R$ be a commutative ring with unity and $A_{i}$ be a prime ideal of $R$ where $i \in I$, an arbitrary indexing set. If $D$ is an ultrafilter on $I$, then the filtered intersection $A$ $=\prod A_{i} / D$ is also a prime ideal of $R$.
Proof. First, assume $I$ is finite and let $D$ be an ultrafilter on $I$. From Lemma 1.5 and Lemma $1.2, D$ is a singleton. This implies that the base element $P\{\alpha\}$ for some $\alpha \in I$. Applying Corollary 2.3, we have that $A=$ $\cap_{i \in P} A_{i}=A_{\alpha}$. Thus, $A_{\alpha}=A$ is a prime ideal since $A_{\alpha}$ is a prime ideal by assumption.

Second, assume $I$ is infinite. The case where $D$ is a singleton is proven in the finite case above. From Corollary 1.6, we only need to consider when every element of $D$ has infinite order. Let $a b=c \in A$ and let $S=\left\{i \mid c \in A_{i}\right\}$. By the definition of filtered intersection, $S \in D$. Define $U=\{j \mid$ $\left.c \in A_{j}\right\}$ and $V=\left\{j \mid c \in A_{j}\right\}$. Since $A_{i}$ is prime for every $i \in S$, either $a \in A_{i}$ or $b \in A_{i}$ for every $i \in S$. This implies $U \cup V=S$.

Assume the $U, V \notin D$, i.e., $a, b \notin A$. Since $D$ is an ultrafilter, we have that $(I \backslash U)$, $(I \mid V) \in D$ and $(I \mid U) \cap(I \mid V) \in D$. One can easily verify $(I \backslash U) \cap(I \mid V)=I \backslash(U \cup V)$ $=I \backslash S \in D$. This is a contradiction of the definition of $D$ being a filter. Therefore, $U \in$ $D$ or $V \in D$ implying that $a \in A$ or $b \in A$. This implies that $A$ is a prime ideal of $R$.

Now we move to a greater generalization of ideals, the module. Recall that an abelian group under addition, $M$, is an $R$-module if there exists a function $f: R \times M$ $\rightarrow M$, denoted $(r, m) \rightarrow r m$ such that (i) $r m$ $\in M$, (ii) $r(m+n)=r m+r n$, and (iii) $(r s) m$ $=r(s m)$. Not surprisingly the filtered intersection of submodules is a submodule.

Theorem 2.9. Let $R$ be a ring and $M$ be an $R$-module. If $M_{i}$ is a submodule for every $i \in$ $I$ and $D$ is a filter over $I$, then $N=\prod M_{i} / D$ is a submodule of $M$.

Proof. Since $M$ is abelian, $N \subseteq M$ is abelian. Let $m \in N$. This yields $S=\{i \mid m \in$ $\left.M_{i}\right\} \in D$. If $m \in M_{i}$, then $r m \in M_{i}$ for every $r$ $\in R$ and $\left\{i \mid r m \in M_{i}\right\} \supseteq S$. This implies $r m$ $\in N$. Properties (ii) and (iii) of a submodule follow similarly. If $m \in M_{i}$, then there exists $-m \in M_{i}$ such that $m+(-m)=0$. Here $\{i \mid$ $\left.(-m) \in M_{i}\right\}=\left\{i \mid m \in M_{i}\right\} \in D$, and if $m \in N$, 
then $(-\mathrm{m}) \in N$. Lastly, since $\left\{i \mid 0 \in M_{i}\right\}=I$ $\in D, 0 \in N$. Altogether, $N \triangleleft M$ and $N$ is a submodule of $M$.

Groups are also very familiar to undergraduate algebraists. The most similar property of ring ideals and modules in groups is that of a normal subgroup. With a similar proof, we find that normal subgroups are preserved under a filtered intersection.

Theorem 2.10. Let $G$ be a group and $H_{i}$ $\triangleleft G$ for all $i \in I$, an arbitrary indexing set. If $D$ is a filter on $I$, then $H=\prod H_{i} / D \triangleleft G$.

Proof. Let $h \in H$ and define $S=\{i \in I \mid h \in$ $\left.H_{i}\right\}$. This yields by the definition of a filtered intersection that $S \in D$. Fix $i$, and since every $H_{i} \triangleleft G$, aha $^{-1} \in H_{i}$ for any $a \in G$. Now define $T_{a}=\left\{j \in I \mid\right.$ aha $\left.^{-1} \in H_{j}\right\}$ where $a$ is fixed. We see that $S \subseteq T_{\alpha}$, and this, combined with part (3) of the definition of a filter, $T_{a} \in D$ for every $a \in G$ and $H$ is normal in $G$.

Since every $H_{i} \triangleleft G, H_{i}$ is a subgroup. Primarily, $e \in H_{i}$ for every $i$, so $e$ $\in H \neq \varnothing$. Secondly, for every $h \in H_{i}, h^{-1} \in$ $H_{i}$ and $\left\{i \in I \mid h^{-1} \in H_{i}\right\}=S \in D$. This yields, if $h \in H$, then $h^{-1} \in H$. We note that associativity is inherited from the group $G$. Therefore $H \triangleleft G$.

Corollary 2.11. Let $\mathrm{G}$ be a group. If $\mathrm{H}, \mathrm{K}$ $\triangleleft G$ and $H \cap K \neq \varnothing$, then $H \cap K \triangleleft G$.

Orderings of sets are merely sets, so we may also take a filtered intersection of them. We will consider several different ways to filter elements and orderings of sets using filtered intersections. Remember that if $a<b$ in a set $S$ with relation $<$, then we write $\langle a, b\rangle \in\langle S,<\rangle$. In Lemma 2.12 we consider the filtered intersection of the sets $\left\langle S,<_{i}\right\rangle$ where $i \in I$, some indexing set. We say that $\langle S,\langle\rangle$ is an ordered set if $a, b \in I$, then (i) $a$ not $<a$, (ii) if $a<b$ and $b<c$, then $a<c$, and (iii) $a=b, a<b$, or $b<a$. If we have several relations on sets $\left\langle S_{i},<_{i}\right\rangle$ where $i \in I$, we say they are non-compromising if a $\neq b$ and $\langle a, b\rangle \in\left\langle S_{i},<_{i}\right\rangle$, then $\langle b, a\rangle \notin\left\langle S_{j},<_{j}\right\rangle$ for all $j \neq i$.

Lemma 2.12. Let $I$ an arbitrary indexing set and $\left\langle S_{i},<_{i}\right\rangle$ for all $i \in I$ be an ordered set. If all the relations are non-compromising and $\left\langle S,\langle\rangle=\Pi\left\langle S_{i},<_{i}\right\rangle / D \neq \varnothing\right.$, then $S$ is an ordered set under the relation $<$ for any for any filter $D$ on $I$.

Proof. By assumption, $S \neq \varnothing$. Let $a, b \in$ $S$ and define $T_{a}=\left\{i \mid a \in S_{i}\right\}$ and $T_{b}=\{i \mid b \in$ $\left.S_{i}\right\}$. Here $a, b \in S$ implies $T_{a}, T_{b} \in D$. Moreover, $\varnothing \neq T_{a} \cap T_{b} \in D$ implying $\{i \mid a, b$ $\in S_{i\}}=T_{a, b} \in D$. For all $i \in T_{a, b}$ we have $a<_{i} b, a=b$, or $b<_{i} a$. Since $T_{a, b} \in D, a<b$, $a=b$, or $b<a$ since the relations are noncompromising. Thus, $S$ is an ordered set under the relation $<$.

A similar result, with a twist, follows if we consider the filtered intersection of the sets $S_{i}$ first and then consider the filtered intersection of the relations $<_{i}$, denoted $\prod\left\langle S_{i} / D,<_{i} / D\right\rangle$. We say that $\langle a, b\rangle \in$ $\prod\left\langle S_{i} / D,<_{i} / D\right\rangle$ if $a, b \in S_{i} / D$ and $\{i \in I \mid$ $\left.\langle a, b\rangle \in\left\langle S_{i},<_{i}\right\rangle\right\} \in D$. Theorem 2.13 shows that ultrafilter is sufficient for the filtered intersection of ordered sets to be an ordered set.

Theorem 2.13. Let $I$ be an arbitrary indexing set and $\left\langle S_{i},<_{i}\right\rangle$ be an ordered set for every $i \in I$. Let $D$ be an ultrafilter on $I$ and let $\langle a, b\rangle \in\left\langle\prod S_{i} / D,<_{i} / D\right\rangle=\langle S,<\rangle$ if $\left\{i \mid a<_{i} b\right\} \in D$ and $a, b \in \prod S_{i} / D$. Then $<$ is an ordering of the set $S$.

Proof. Let $S \neq \varnothing$ and $a, b \in S$, where $a \neq b$. Note that $\varnothing=\left\{i \mid a<_{i} b\right\} \cap\left\{i \mid b<_{i}\right.$ a $\}$ not $\in D$ because each $S_{i}$ is ordered. Thus, the relation is well defined. Since every set is ordered, we have the following results. $\varnothing=$ $\left\{i \mid a<_{i} a\right\}$ not $\in D$, which implies for any $a \in$ $S$ we do not have $a<a$. Given $a<b$ and $b<$ $c$, we have $\left\{i \mid a<_{i} b\right\},\left\{i \mid b<_{i} c\right\} \in D$. This implies $\left\{i \mid a<_{i} b\right\} \cap\left\{i \mid b<_{i} c\right\} \in D$, and $a<$ c. 
Assume $a$ not $<b$ and $b$ not $<a$. Then $\left\{i \mid a<_{1} b\right\}, \quad\left\{i \mid b<_{i} c\right\} \notin D$ implying $I$ $\backslash\left\{i \mid a<_{i} b\right\}, I \backslash\left\{i \mid b<_{i} a\right\} \in D$. This yields $I$ $\backslash\left\{i \mid a<_{i} b\right\} \cup I \backslash\left\{i \mid b<_{i} a\right\}=I \backslash\left\{i \mid a<_{i} b\right\}$ $\cap I \backslash\left\{i \mid b<_{i} a\right\} \in D$. However, $\left\{i \mid a \in S_{i}\right\}$, $\left\{i \mid b \in S_{i}\right\} \in D$ implies $\left\{i \mid a \in S_{i}\right\} \cap\{i \mid b \in$ $\left.S_{i}\right\} \in D$ and $\varnothing=\left(I \backslash \quad\left(\left\{i \mid a<_{i} b\right\} \cup\left\{i \mid b<_{i}\right.\right.\right.$ a\})) $\cap\left\{\left\{i \mid a \in s_{i}\right\} \cap\left\{i \mid b \in S_{i}\right\}\right) \in D\left({ }^{*}\right)$. By the definition of a filter, $\varnothing$ not $\in D$. Thus, $\left({ }^{*}\right)$ is a contradiction and yields that for every $a, b \in S$ that $a<b$, $a=b$, or $b<a$. Therefore, $<$ is an ordering on $S$.

We could limit ourselves to just one particular set and several orderings on the set. Theorem 2.13 implies the following corollary where we only consider one particular set and the filtered intersections of possible ordering of the set.

Corollary 2.14. Let $I$ be an arbitrary indexing set and $\left\langle S,<_{i}\right\rangle$ be an ordered set for every $i \in I$. Let $D$ be an ultrafilter on $I$ and let $\langle a, b\rangle \in\left\langle S,<_{i} / D\right\rangle=\langle S,<\rangle$ if $\left\{i \mid a<_{i}\right.$ $b\} \in D$. Then $<$ is an ordering of the set $S$.

Example 2.15 shows that neither weakening the condition of $D$ being an ultrafilter in theorem 2.13 nor weakening the non-compromising restriction in Lemma 2.12 fails to yield similar results. It also shows a similar necessity of the ultrafilter property in Corollary 2.14.

Example 2.15. Consider the sets $\langle\mathrm{Z},<\rangle$ and $\left\langle\mathrm{Z},<^{*}\right\rangle$, where $<^{*}$ is the reverse ordering, indexed as $\alpha, \beta$ respectively. Then $\prod S_{i} / D$ $=\mathrm{Z}$ no matter the filter; however, given $D=$ $\{\alpha, \beta\}$, no two different elements are comparable. Clearly $3,5 \in Z$, but $\{i \mid 3<5\}$ $=\{\alpha\}$ not $\in D$ and $\left\{i \mid 5<^{*} 3\right\}=\{\beta\}$ not $\in D$. Therefore, the filtered intersection $\left\langle\prod S_{i} / D\right.$, $<_{i}|D\rangle=\left\langle Z,<_{i} \mid D\right\rangle=\varnothing$, i.e., the filtered intersection of the relation is not ordered. Note that the filtered intersection of the sets is non-empty and only the set of the orderings is empty.

Partially ordered sets are a weaker condition on a set with a relation. We say a set $S$ is partially ordered under the relation $<$ if the relation satisfies conditions (i) and (ii) in the definition of an ordered set, but does not satisfy condition (iii). A very simple corollary follows for partially ordered sets where the condition of an ultrafilter is unnecessary.

Corollary 2.16. Let $I$ be an arbitrary indexing set and $\left\langle S,<_{i}\right\rangle$ be an ordered set for every $i \in I$. Let $D$ be a filter on I and let $\langle a, b\rangle \in\left\langle\prod S_{i} / D,<_{i} / D\right\rangle=\langle S,<\rangle$ if and only if $\left\{i \mid a<_{i} b\right\} \in D$. Then $<$ is a partial ordering of the set $S$.

Proof. See the first paragraph of the proof of Theorem 2.13.

The final significant ordering property yet to be discussed is that of a set being well-ordered. We remind the reader that if a set is well-ordered then every nonempty subset has a least element. There exists sufficient properties to have ordered and partially ordered sets under the filtered intersection, but no sufficient property has been found to have a well-ordered set. The following example shows that even a filter with elements of infinite order save one element is not sufficient to yield a wellordered set.

Example 2.17. Let $\mathrm{Q}_{\mathrm{n}}=\{m / n \mid m, n \in \mathrm{N}$ and $0<m \leq n\}$ and let $I=\mathrm{N}$. The set $D=$ $\{M \mid M \supseteq(\mathrm{n}), \mathrm{n} \in \mathrm{N}\}$ is a filter on $I$ (the proof is left to the reader). We note that $D$ is not an ultrafilter as $U=\{p \in N \mid p$ is prime $\}$ not $\supseteq(n)$ and $\mathrm{N} \backslash U=\{c \in \mathrm{N} \mid c$ is not prime $\}$ not $\supseteq(n)$ for every $n \in \mathrm{N}$, so $U, \mathrm{~N} \backslash U \notin D$. Consider $s=p / q \in Q, 0<s \leq 1$, and $p, q \in$ $\mathrm{Z}$ with $\operatorname{gcd}(p, q)=1$. Then $s \in \mathrm{Q}_{\mathrm{y}}$ for all $q \mid y$. If $s \in Q_{z}$, then $p / q=s=x / z$ for some $x, z \in \mathrm{N}$. Thus, $q \mid p z$, and since $q$ not $|p, q| z$. This implies that $=\left\{i \mid s \in Q_{i}\right\}=$ $(q) \in D$ and $s \in \prod Q_{n} / D$. Thus $\prod Q_{n} / D=\{l$ $<s \in Q \mid 0<s \leq 1\}=Q \cap(0,1]$. If we use the usual $<$ relation on the rationals, we see that $\prod Q_{n} / D=Q \cap(0,1]$ fails to have a least element. In fact, any subset $A=\{I<s \mid$ $I \in(0,1)$ and $I \notin Q\}$ will fail to have a least element since $Q$ is a dense set under the < relation. However, each $Q_{n}$ is finite and 
ordered implying it is well-ordered also. This example shows that an ultrafilter is not a necessary condition for a filtered intersection of ordered sets to be ordered.

\section{FILTERED PRODUCTS}

The purpose of the filter changes in a filtered product. No longer are we using it to select elements to be in a final set; rather, we use it to create equivalence classes of all the elements of a Cartesian product.

Definition 3.1. Let $I$ be an index, $D$ be a filter on $I$, and $A_{i}$ be a set for any $i \in I$. If we give the Cartesian product $\prod_{i \in I} A_{i}$ the equivalence relation $a \sim b$ if and only if $\left\{i \mid a_{i}\right.$ $\left.=b_{i}\right\} \in D$, then we call the set of equivalence classes the filtered product of the $A_{i}$ 's with respect to $D$, denoted $\prod A_{i} / \sim D$.

As good mathematicians, we cannot assume that the definition above is an equivalence relation: We must prove it to be true.

Lemma 3.2. The relation in Definition 3.1 is an equivalence relation on $A=\prod_{i \in I} A_{i}$.

Proof. Let $a=\left(a_{1}, a_{2}, \ldots\right) \in A$ and $\left(b_{1}\right.$, $\left.b_{2}, \ldots\right) \in A$. Clearly $\left\{i \mid a_{i}=b_{i}\right\}=I \in D$ and $a$ $\sim a$. Let $a \sim b$, so $\left\{i \mid a_{i}=b_{i}\right\} \in D$ implies $\left\{i \mid b_{i}=a_{i}\right\} \in D$ and $b \sim a$. Let $a \sim b$ and $b \sim$ $c$, so $\left\{i \mid a_{i}=b_{i}\right\},\left\{i \mid b_{i}=c_{i}\right\} \in D$. By definition of a filter, $\varnothing \neq\left\{i \mid a_{i}=c_{i}\right\} \supseteq\left\{i \mid a_{i}=b_{i}\right\} \cap\left\{i \mid b_{i}\right.$ $\left.=c_{i}\right\} \in D$ and implies $a \sim c$. Therefore, the relation is an equivalence on $A=\prod_{i \in I} A_{i}$.

One can easily verify that $\prod R_{i} / \sim D$ is a ring. Again, we first investigate the role of ideals and find similar results as those concerning filtered intersections.

Theorem 3.3. The filtered product of ideals is an ideal. That is $\prod A_{i} / \sim D$ is an ideal of $\prod R_{i} / \sim D$.

Proof. Let $A_{i}$ be an ideal of the ring $R_{i}$ for all $i \in I$, some indexing set. Since $0 \in A_{i}$ for every $i \in I,(0,0, \ldots) \in \prod A_{i} / \sim D$.
Instead of considering the congruence classes of $\prod A_{i} / \sim D$, namely, [a], $[b]$, let us consider the individual elements. Then we show that the result follows for the congruence class. Let $a, b \in \prod A_{i} / \sim D$, where $a=\left(a_{1}, a_{2}, \ldots\right)$ and $b=\left(b_{1}, b_{2}, \ldots\right)$. This implies that $\left\{i \mid a_{i} \in A_{i}\right\},\left\{i \mid b_{i} \in A_{i}\right\} \in D$. Therefore, $\left\{i \mid a_{i}-b_{i} \in A_{i}\right\} \supset\left\{i \mid a_{i} \in A_{i}\right\} \cap$ $\left\{i \mid b_{i} \in A_{i}\right\}$, so $\left\{i \mid a_{i}-b_{i} \in A_{i}\right\} \in D$. Thus, $a$ $-b \in \prod A_{i} / \sim D$. Let $r=\left(r_{1}, r_{2}, \ldots\right)$ and consider $r a=\left(r_{1} a_{1}, r_{2} a_{2}, \ldots\right)$. Here, if $a_{i} \in A_{i}$, then $r_{i} a_{i} \in A_{i}$. This implies that $\left\{i \mid r_{i} a_{i} \in A_{i}\right\}$ $\supset\left\{i \mid a_{i} \in A_{i}\right\}$. Thus, $\left\{i \mid r_{i} a_{i} \in A_{i}\right\} \in D$ and hence $r a \in \prod A_{i} / \sim D$.

Let $[a],[b] \in \prod A_{i} / \sim D$. For every $a$ $\in[a]$ and $b \in[b], a-b \in \prod A_{i} / \sim D$ from above. This yields that $[a]-[b] \in \prod A_{i} / \sim D$. Similarly, we have that $[r][a] \in \prod A_{i} / \sim D$. Therefore, $\prod A_{i} / \sim D$ is an ideal of $\prod R_{i} / \sim D$.

Like filtered intersections, filtered products do not necessarily preserve the property of prime ideals.

Example 3.4. Consider $\left\{\mathrm{Z}_{6}, \mathrm{Z}_{6}\right\}$ indexed as $\{\alpha, \beta\}=I$. If we let $A_{\alpha}=(2)$ and $A_{\beta}=(3)$, then our $A_{i}$ 's are prime ideals. Let $D=\{\alpha$, $\beta$, a filter on $I$. We note that $(1,2)(3,2)=$ $(3,2) \in \Pi A_{i} / \sim D$. However, $(1,2),(3,1) \notin$ $\prod A_{i} / \sim D$. Therefore, the ideal product is no longer prime.

\section{IDEAL INTERSECTIONS}

As noted, a filter is simply a subset of the power set of a given set that satisfies certain properties. When we consider the compliment of the filter, called an ideal, we find similar properties with one notable difference.

Let $X$ be a nonempty set and let $D$ be a filter on $X$. From [2], we say that $F=$ $P(X) \backslash D$, the compliment of the filter $D$, is an ideal on $X$.

Definition 4.1. Let $X$ be a nonempty set. $A$ set system $F \subset P(X)$ is said to be an ideal on $X$ if $F$ has the following properties:

(i) $X \notin F$, 
(ii) If $A, B \in F$, then $A \cup B \in F$, and

(iii) If $A \in F$ and $B \subset A$, then $B \in F$.

The set system $F \subset P(X)$ is called a prime ideal on $X$ if it is an ideal and there is no ideal $F^{\prime} \subset P(X)$ on $X$ for which $F$ not $\subset F^{\prime}$.

Complimenting property (iii) of a filter, property (iii) of an ideal is known as being "closed downward." The following results can be found in [2] or proven by the reader.

Lemma 4.2. Let $X$ be a nonempty set. Then $F$ is a prime ideal on $X$, the $D=P(X) \mid F$ is a filter on $X$.

Lemma 4.3. Let $X$ be a nonempty set. Then $F$ is a prime ideal on $X$ if and only if $D$ $=P(X) \backslash F$ is an ultrafilter on $X$.

We define ideal intersections in the expected manner, using the ideal structure in place in the former filter structure.

Definition 4.4. Let be an index $I, F$ be an ideal on $I$, and $C_{i}$ be a set for every $i \in I$. Then $\left\{a \mid\left\{i \mid a \in C_{i}\right\} \in F\right\}$ is called the ideal intersection of the $C_{i}$ with respect to $F$, denoted $\Pi C_{i} / F$.

Unsurprisingly, there is a direct correlation between ideal intersections and filtered intersections. Theorem 4.5 shows that the ideal intersection of a set $X$ over the ideal $I$ is completely determined by the filtered intersection of $X$ over the filter $F=$ $P(X) \backslash I$ when $F$ is a principal filter.

Theorem 4.5. Let $X$ be a nonempty set and let $F=\{B \in P(X) \mid B \supseteq P\}$ for some fixed $P$ $\in P(X)$ with finite cardinality. Let $C_{i}$ be a nonempty set for every $i \in X$. The ideal intersection with respect to $F$ will be $C=$ $\prod C_{i} / F=\left(\cap_{i \in I} C_{i}\right) \mid\left(\cap_{i \in P} C_{i}\right)$.

Proof. Define $D=P(X) \backslash F=\{A \in P(X) \mid A \supseteq$ $P$, a filter. From Theorem 2.2, we have that the filtered intersection with respect to $D$ is $\cap_{i \in P} C_{i}$. If $a \in C_{i}$ for every $i \in P$, then $\mathrm{S}=$ $\left\{i \mid a \in C_{i}\right\} \supseteq P$. Thus, $S \notin F$ and $a \notin C$. If $b \notin \cap_{i \in P} C_{i}$, then there exists a $j \in P$ such that $b \notin C_{j}$. This implies that $T=\left\{i \mid b \in C_{i}\right\}$ not $\supseteq P$, so $T \in F$ and $b \in C$. Therefore, $C$ $=\prod C_{i} / F=\left(\cap_{i \in I} C_{i}\right) \backslash\left(\cap_{i \in P} C_{i}\right)$.

Theorem 4.5 can easily be extended to all ideal and filtered intersection so long as $F=P(X) \backslash Y$. Given that $F \cup(P(X) \backslash F)=$ $\mathrm{D} \cup(P(X) \backslash D)=P(X)$ for any ideal $F$ and filter $D$ on $X$, one would expect the following theorem and it can be easily verified.

Theorem 4.6. Let $X$ be a nonempty set. For any filter $D$ and ideal $F=(P(X) \backslash D)$ on $X$, we have that $\prod A_{i} / F \cup \prod A_{i} /(P(X) \backslash F)=$ $\prod A_{i} / D \cup \prod A_{i} \quad /(P(X) \backslash D)=\cap_{i \in X} A_{i}$. Moreover, $\prod A_{i} / F=\prod A_{i} /(P(X) \backslash D), \prod A_{i} / D$ $=\prod A_{i} /(P(X) \backslash F)$, and $\prod A_{i} / F \cap \prod A_{i} / D=$ $\varnothing$.

Unlike filtered intersections, ideal intersections do not have to preserve the property of ring ideals. This is shown in Example 4.7 and generalized in theorem 4.8 .

Example 4.7. Consider (2), (3) $\subset \mathrm{Z}$ and index them as $\{\alpha, \beta\}=I$ respectively. If we use the ideal $\mathrm{F}=\{\{\alpha\},\{\beta\}, \varnothing\}$ on $I$, then the ideal intersection is (2) $\cup(3)=\{a \in Z|2| a$ or $3 \mid a$ and 6 not $\mid a\}$. This implies that 2, 3 $\in C$. However, 2 not $\mid 1$ and 3 not $\mid 1$, but $3-2=1 \notin C$. Therefore, $C$ is not an ideal.

Theorem 4.8. Let $R$ be a commutative ring with unity and $A_{i}$ be an ideal of $R$ where $i \in$ $I$, an arbitrary indexing set. If $F$ is an ideal on $I$, then the ideal intersection $C=\prod A_{i} / F$ will not be an ideal of $R$.

Proof. Let $D=P(I) \backslash F$. Consider $a \in A=$ $\prod A_{i} / D$ and $c \in C=\prod A_{i} / F$. From Theorem 2.5, we have that $A$ is an ideal and ac $\in A$. This and the last statement of Theorem 4.6 imply that ac $\notin C$. Therefore, $C$ is not an ideal.

Although the filtered intersection may not be an ideal, the property of being prime is conserved. 
Lemma 4.9. Let $R$ be a commutative ring with unity and $P_{i}$ be a prime ideal of $R$ where $i \in I$, an arbitrary indexing set. If $F$ is an ideal on $I$, then the ideal intersection $P=$ $\prod P_{i} / F$ will retain the prime property, i.e., if $a b \in P$, then $a \in P$ or $b \in P$ (particularly we find that $a, b \in P$ ).

Proof. Let $c \in P$ and let $a b=c$. Define $S=$ $\left\{i \mid c \in P_{i}\right\}$. From the definition of an ideal intersection $S \in F$. If $a \in P_{j}$, the $c=a b \in$ $P_{j}$. Thus, $\left\{j \mid a \in P_{j}\right\} \subset S$. The last condition of the definition of an ideal gives $\left\{j \mid a \in P_{j}\right\}$ $\in F$. This implies $a \in P$. Similarly, $b \in P$.

Many more questions remain to be explored in ideal intersections and products. Most interesting is to consider similar inputs, like sub-modules, under filtered intersections and ideal intersections. As discovered, some of the results correlate nicely between filtered and ideal intersections, but others are wholly opposite the result of the other. Similarly, these properties could be explored further in filtered products and ideal products. Lastly, many other properties of sets could be explored under intersections and products. As stated in the beginning, many results for filtered intersections, despite one's efforts, may be corollaries to Łos' Theorem.

\section{REFERENCES}

1. J.A. Gallian, Contemporary Abstract Algebra (4 $4^{\text {th }}$ edition). (Houghton Mifflin Company, New York, 1998).

2. A. Hajanl and P. Hamburger, Set Theory. (Cambridge University Press, Cambridge CB2 2RU, U.K.).

3. M.D. Morley, ed., Studies in Model Theory. (The Mathematical Association of America, Washington DC, 1973).

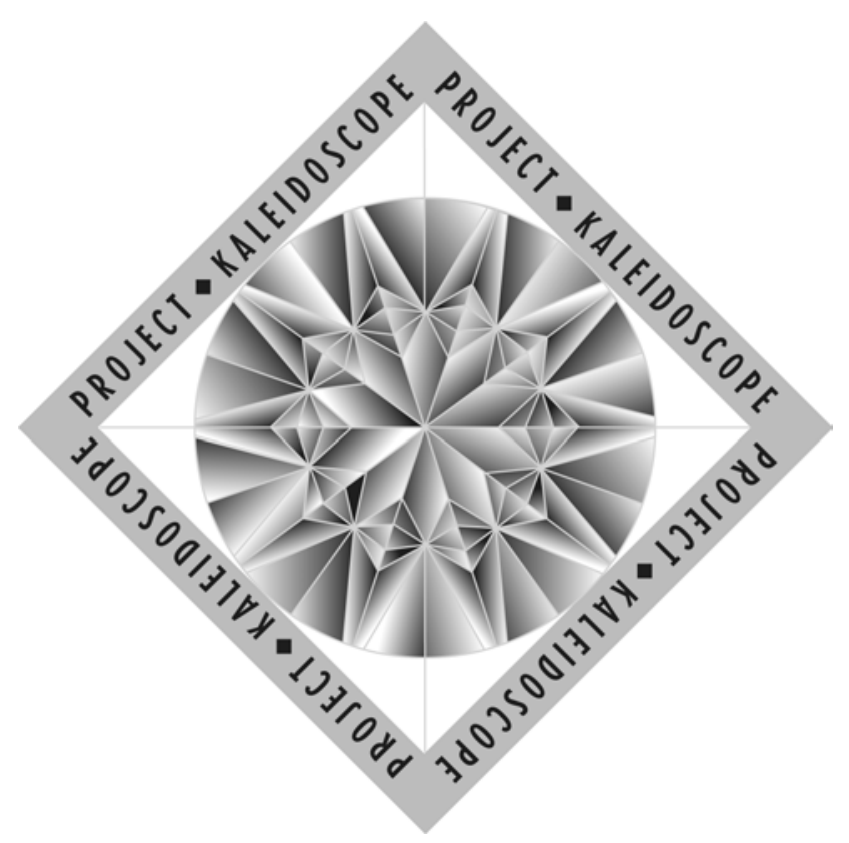

\section{Project Kaleidoscope www.pkal.org}

